\title{
A Uniform Heating Technique for Cavity in Volatile Organic Compound (VOC) Removal System Using Slotted Waveguide Array
}

\author{
Taewoo $\mathrm{Yu}^{1} \cdot$ Hyunwook Lee ${ }^{2} \cdot$ Sang-Jun Park $^{2} \cdot$ Sangwook Nam ${ }^{1, *}$
}

\begin{abstract}
In this study, two types of slotted waveguide are designed in the frequency of $2.45 \mathrm{GHz}$ to improve the microwave heating uniformity of a quadrangular prism-shaped cavity in a volatile organic compound (VOC) removal system. Both types adopt the equivalent circuit approach used for a waveguide slot array antenna. The difference between the two types is the slot impedance extraction method of the waveguide slot array: one calculates the impedance taking the cavity structure into account and the other finds it in free space. Both methods show that the heating uniformity is improved by $52 \%$ compared with that of the conventional horn-type feeding structure system according to the simulation results. Even though there is no difference in the heating uniformity between the two models, it is confirmed that the slotted waveguide array feeding model designed by using the impedance data of the slot incorporating the cavity $\left(\mathrm{SAWFM}_{\text {cavity }}\right)$ has about $6.35 \mathrm{~dB}$ better impedance matching characteristics than the other model designed by extracting the impedance data of the slot in free space $\left(\mathrm{SAWFM}_{\text {free }}\right)$. Also, it is found that the $\mathrm{SAWFM}_{\text {cavity }}$ shows more stable impedance characteristics with respect to the loading condition than the SAWFM free. Therefore, it is concluded that the impedance of the slot should be extracted taking the cavity into account for the design of the slotted waveguide feeding structure since it improves the reflection characteristic as well as the heating uniformity compared with the horn-type feeding structure.
\end{abstract}

Key Words: Cavity, Heating Uniformity, Impedance Matching, Power Loss Density Distribution, Slotted Waveguide.

\section{INTRODUCTION}

Microwave heating has been used for a long time in commercial and industrial applications owing to its heating efficiency. A typical example of a heating method using microwaves is a microwave oven at home. It is also used industrially in various fields such as soil moisture removal, volatile organic compound (VOC) removal, disinfection, and sterilization [1-3].

VOCs such as toluene and xylene inevitably occur in many industrial facilities, including semiconductor processing plants, refineries, and shipyards [4]. To prevent environmental pollution and human hazards, VOCs should be filtered by VOC filters. The confinement process of VOCs through filters is called adsorption. Owing to their volatility, the filters used for VOC adsorption can be recycled through microwave heating, and the desorbed VOCs can be separated by an air intake system. This process is called desorption. The adsorption and desorption processes are sequentially performed in separate spaces. To regenerate the VOC filter well, high energy efficiency and heating uniformity are required during desorption.

Manuscript received July 7, 2020 ; Revised October 26, 2020 ; Accepted December 8, 2020. (ID No. 20200707-099J)

${ }^{1}$ Department of Electronic and Computer Engineering, INMC, Seoul National University, Seoul, Korea.

${ }^{2}$ Department of MW Project Team, ECOPRO Co. Ltd., Cheongju, Korea.

*Corresponding Author: Sangwook Nam (e-mail: snam@snu.ac.kr)

This is an Open-Access article distributed under the terms of the Creative Commons Attribution Non-Commercial License (http://creativecommons.org/licenses/by-nc/4.0) which permits unrestricted non-commercial use, distribution, and reproduction in any medium, provided the original work is properly cited.

(c) Copyright The Korean Institute of Electromagnetic Engineering and Science. All Rights Reserved. 
Since most microwave heating systems are high-power applications, the shielding is very important, and thus, a cavity structure is frequently used. In addition, to avoid dielectric breakdown, the microwave feeding structure often uses a waveguide aperture type composed of a single conductor. For example, a VOC removal facility using a cavity fed by a horn-type microwave feeding structure is suggested [5].

In general, much care is needed for the design of the feeding structure of a cavity since many modes exist inside the cavity as determined by the cavity structure, and the excitation of the mode is dependent on the feeding structure. Many research studies related to this have been actively conducted [6-12]. The optimization process for improving the electric field's uniformity by analyzing the modes existing in the cavity and expressing the electric field inside the cavity in a linear combination of the modes has been presented [6]. However, modal analysis in a cavity and finding the feeding structure are very difficult because the cavity structures have different characteristics. Another method proposed for improving the uniformity is to use multiple microwave sources with sequential feeding [7]. However, using multiple sources has the disadvantages of system complexity and higher cost. The other method of increasing the uniformity is to employ a mode stirrer to mix multiple electromagnetic modes in the cavity, and parametric studies with a genetic algorithm for a dielectric multilayer in a rectangular cavity were suggested $[8,9]$. Nevertheless, the mode stirrer itself is an extra structure that makes the system complicated, and the parametric studies with a genetic algorithm are also difficult to analyze.

There are studies in which dielectric slabs are uniformly heated using a slotted waveguide structure, and studies in which the heating uniformity is improved by using multiple slotted waveguides in a rectangular cavity [10-12]. Unlike the methods introduced earlier, these feeding structures can have the effect of generating multiple microwave sources with a single excitation port in a simple design method. However, the technique was used for heating a dielectric slab and the rectangular cavity only.

In this study, two types of slotted waveguide feeding structures are designed for the uniform heating of a VOC absorbent material in a quadrangular prism-shaped cavity used in a VOC removal facility. This paper is organized as follows. The entire VOC removal system, the cavity structure, and the basic horntype feeding structure are introduced in Section II. In Section III, the basic theory of waveguide slot and the design method of the two types of slotted waveguide feeding structures are presented. In Section IV, the figure of merit of heating uniformity used in this study is presented, and the comparison result of the heating uniformity performance among the horn-type and the two types of slotted waveguide feeding structures is introduced. The impedance matching characteristics are compared and the load sensitivity is discussed in Section V, followed by the conclu- sion in Section VI.

\section{ENTIRE VOC REMOVAL SYSTEM AND HORN-TYPE}

\section{FEEDING STRUCTURE}

Fig. 1(a) shows the entire VOC removal system [4]. The total structure is a large metallic cylinder divided into several cylindrical wedge-shaped rooms, and the red part represents the air intake system. Each room is shielded with metal plates, and the upper and lower surfaces are composed of a metal perforated plate, which shields microwaves, but air can be inhaled. A VOC absorbent material (so-called VOC filter) is partially filled in each room, and microwaves are applied to each room to desorb VOC from the filter. The desorbed VOC is removed by the air intake system, and the system is rotated every certain time to remove the desorbed VOC in other spaces. Owing to the air intake system, microwaves cannot be fed from the top and bottom, but from the outer face of the system or sidewall of the cavity. Fig. 1(b) shows the quadrangular prism-shaped cavity structure for uniform heating used in this study, which is actually a piece of 16 room cavities arranged in circular form in the VOC removal system shown in Fig. 1(a). Table 1 shows the geometric parameters of the cavity. The cavity is partially filled with a dielectric material (VOC filter after adsorption) to be heated, the dielectric constant and loss tangent of which are 4.98 and 0.16 , respectively.

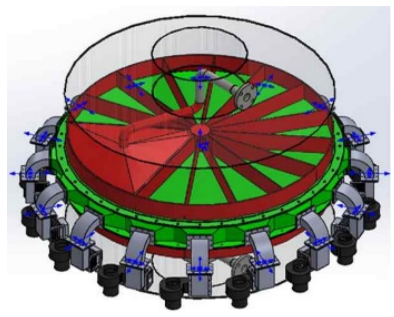

(a)

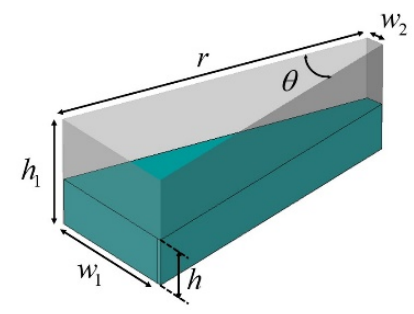

(b)
Fig. 1. (a) Entire VOC removal system. (b) A piece of 16 cavities of the entire system (quadrangular prism-shaped cavity).

Table 1. Geometric parameters of the cavity

\begin{tabular}{lr}
\hline Parameter & Value \\
\hline$r \quad(\mathrm{~mm})$ & 990.6 \\
$h_{1}(\mathrm{~mm})$ & 350.0 \\
$h \quad(\mathrm{~mm})$ & 150.0 \\
$w_{1}(\mathrm{~mm})$ & 448.1 \\
$w_{2}(\mathrm{~mm})$ & 58.2 \\
$\theta \quad\left({ }^{\circ}\right)$ & 22.5 \\
\hline
\end{tabular}


Fig. 2 shows a piece of room cavity with the conventional horn-type feeding structure. In this study, two types of slotted waveguide are designed and attached at the sidewall of the cavity to improve the heating uniformity.

\section{Two Types Of SLOTTED WAVEGUIDE DESIGN}

The microwave source used for heating is a magnetron operating at $2.45 \mathrm{GHz}$. Therefore, the waveguide structure of WR340 (width $=86 \mathrm{~mm}$, height $=43 \mathrm{~mm}$ ) is selected. The first method (hereinafter, $\mathrm{SAWFM}_{\text {free}}$ ) is to design the microwave feeding structure according to the well-known broad-wall resonant waveguide slot array (WSA) antenna design method. This method has been used for the uniform heating of a rectangular cavity [12].

Fig. 3(a) shows a longitudinal slot with an offset on a broad wall of a rectangular waveguide. The slot length is around $\lambda_{0} / 2$ so that the slot shows a resonance characteristic. Specifically, this structure can be modeled as a shunt admittance element [1315]. When the $\mathrm{TE}_{10}$ mode is excited in the waveguide, the normalized shunt admittance value can be calculated as

$$
y=2.09 \frac{\lambda_{g}}{\lambda_{0}} \frac{a}{b} \cos ^{2}\left(\frac{\pi \lambda_{0}}{2 \lambda_{g}}\right) \sin ^{2}\left(\frac{\pi d}{a}\right),
$$

where $\lambda_{g}$ is the guided wavelength in the waveguide [13].

Fig. 4 shows the broad-wall resonant single slot used in this study. Since the admittance value may vary depending on the thickness and width of the slot, a more accurate admittance value can be obtained through full wave simulation. In this study, the

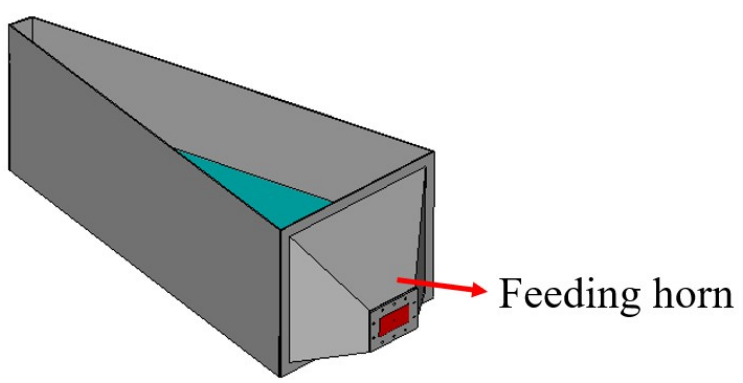

Fig. 2. Horn-type microwave excitation structure for the quadrangular prism-shaped cavity.

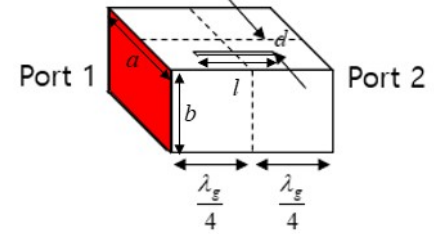

(a)

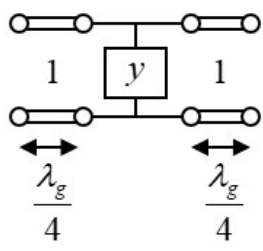

(b)
Fig. 3. (a) Longitudinal slot on a broad wall of waveguide. (b) Equivalent circuit model of Fig. 2(a).

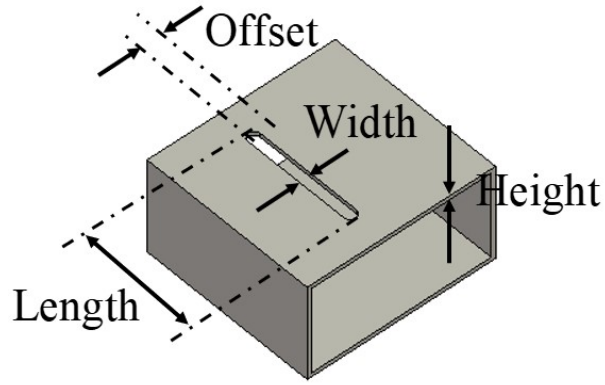

Fig. 4. Broad-wall resonant single slot.

slot width and height, shown in Fig. 4, are fixed to $6 \mathrm{~mm}$ and 5 $\mathrm{mm}$, respectively. The normalized admittance value can be calculated as

$$
y=\frac{\left(\left(1-S_{11}\right)^{2}-S_{21}^{2}\right)}{2 S_{21}},
$$

where the normalization factor is a wave impedance of the $\mathrm{TE}_{10}$ mode in the rectangular waveguide. The scattering parameters used in Eq. (2) are the data obtained by simulating the waveguide slot and de-embedding the $\lambda_{\mathrm{g}} / 4$ waveguide section on each port. The basic WSA antenna design method uses an equivalent circuit approach where the calculated shunt admittance elements representing broad-wall slots are connected by the transmission line representing the waveguide. For broadside radiation, the slot spacing is set to $\lambda_{g} / 2$, and the offset direction of the adjacent slot is opposite to compensate the phase of the $\lambda_{\mathrm{g}} / 2$ waveguide section. The end of the waveguide is short, and the distance from the end to the last slot is $\lambda_{g} / 4$ [13]. Fig. 5 shows the equivalent circuit model of the slotted waveguide structure, and the normalized input admittance can be calculated as

$$
y_{i n}=\sum_{k=1}^{n} y_{k},
$$

where $y_{k}$ is the normalized admittance value of the $k$ th slot. Considering the overall size of the cavity, the total number of slots is set to 10. Following the broad-side radiation WSA antenna design, the normalized shunt conductance and susceptance value are set close to 0.1 and 0 , respectively. The waveguide center is placed at a distance of $235 \mathrm{~mm}$ from the floor of the system to

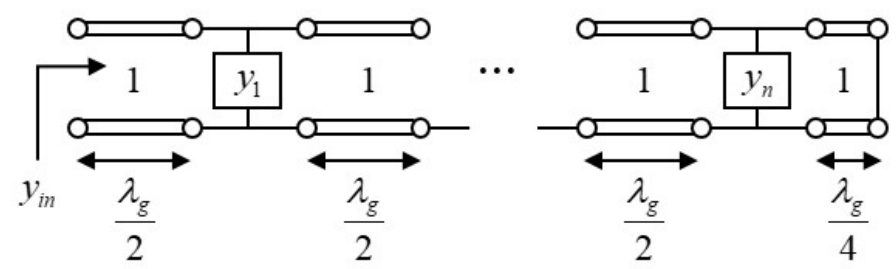

Fig. 5. Equivalent circuit model of slotted waveguide to be used as the feeding structure of the cavity. 
avoid direct contact between the VOC absorbent dielectric and the slot. Fig. 6 shows the entire heating system with the slotted waveguide feeding structure, and Table 2 shows the normalized admittance value of each slot and the length and offset properties. The height of the centerline of the slot array and the position of the first slot (\#1) are fixed to $z=235 \mathrm{~mm}$ and $y_{1}=42.08$ $\mathrm{mm}$, respectively. Also, the quantity of the VOC absorbent material (loading) depends on the operational situation so that the height $b$ is a variable in the range of 130 to $170 \mathrm{~mm}$. The reference $b$ is set to $150 \mathrm{~mm}$, which is an intermediate value of the height range.

For $\mathrm{SAWFM}_{\text {free, }}$ the feeding structure is designed using the resonant broad-side WSA antenna design method in free space. However, as soon as the slotted waveguide structure is attached to the sidewall of the cavity, the calculated admittance property is changed by the cavity structure. After all, in order to find the correct admittance data of the slot attached to the cavity, the admittance properties have to be extracted including the effect of the cavity. The second method (hereinafter, SAWFM $_{\text {cavity) }}$ is to extract admittance data of the slot including the effect of the cavity and to design the slotted waveguide feeding structure in the same way as the approach in $\mathrm{SAWFM}_{\text {free. }}$. In this case, two slots having the same length and offset have different admittances, since the fields excited by the two slots are different depending on the position of the slot inside the cavity. Hence, the position of the slot should be considered as a variable. The height of the centerline of the slot array and the position of the first slot are the same as in the $\mathrm{SAWFM}_{\text {free }}$ case. Fig. 7 shows the extracted normalized admittance data with varying lengths and offsets of all slots. Since there are two variables, namely, the slot's length and offset, the admittance value is calculated by fixing one variable and changing the other variable. Fig. 7(a) and

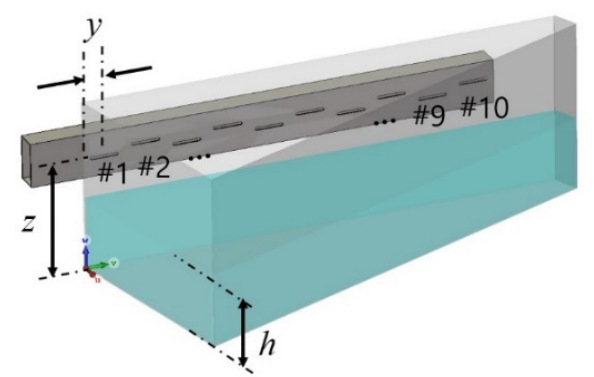

Fig. 6. A 3D model of the system with the slotted waveguide feeding structure: $z=235 \mathrm{~mm}, y_{1}=42.08 \mathrm{~mm}, h=150 \mathrm{~mm}$.

Table 2. Normalized shunt admittance values at $2.45 \mathrm{GHz}$ and slot's properties $\left(\mathrm{SAWFM}_{\text {free }}\right)$

\begin{tabular}{cccc}
\hline \multirow{2}{*}{ Slot } & \multirow{2}{*}{$\begin{array}{c}\text { Normalized } \\
\text { admittance }\end{array}$} & \multicolumn{2}{c}{ Properties $(\mathrm{mm})$} \\
\cline { 3 - 4 } $1-10$ & $0.1026+j 0.0006$ & 8.4 & Offset
\end{tabular}

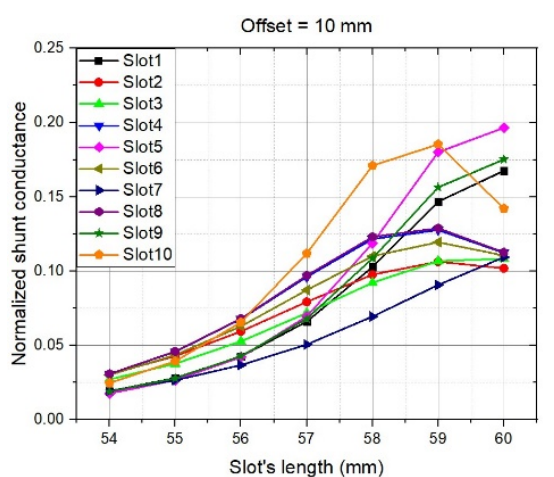

(a)

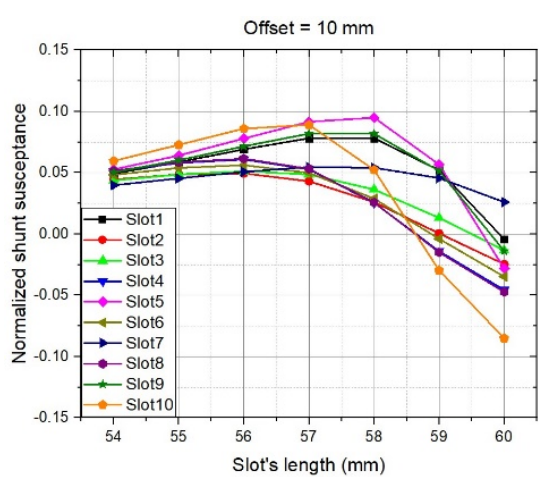

(b)

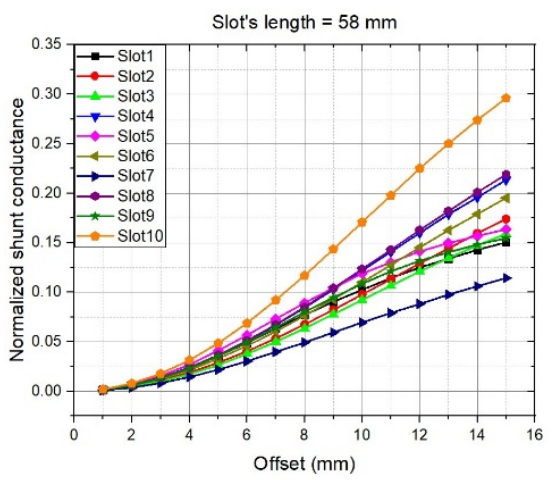

(c)

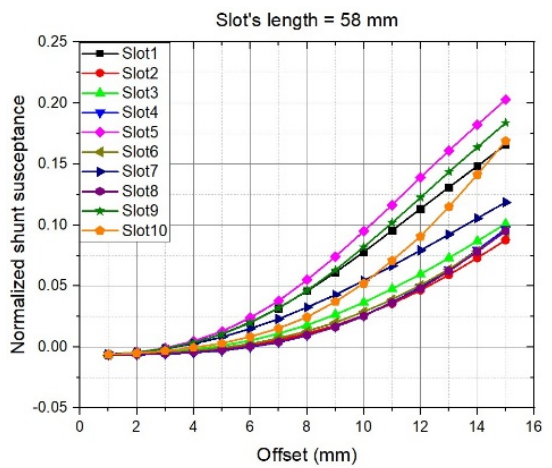

(d)

Fig. 7. Extracted normalized shunt admittance (SAWFM ${ }_{\text {cavity }}$ ): (a) normalized shunt conductance with respect to the slot's length (Offset $=10 \mathrm{~mm}$ ), (b) normalized shunt susceptance with respect to the slot's length (Offset $=10 \mathrm{~mm}$ ), (c) normalized shunt conductance with respect to the slot's offset (Length $=$ $58 \mathrm{~mm}$ ), and (d) normalized shunt susceptance with respect to the slot's offset (Length $=58 \mathrm{~mm}$ ). 
(b) show the normalized shunt conductance and susceptance, respectively, according to the length change when the slot's offset is fixed at $10 \mathrm{~mm}$. Fig. 7(c) and (d) show the normalized shunt conductance and susceptance, respectively, according to the offset change when the slot's length is fixed at $58 \mathrm{~mm}$. Notice that the admittance values are quite different depending on the slot's position as the slot length or offset is large. On the basis of the data in Fig. 7, a slight tuning is performed to match the normalized shunt admittance value of each slot similar to SAWFM $_{\text {free. }}$ Table 3 shows the derived slot's properties of SAWFM $_{\text {carity. }}$

\section{UNIFORM HEATING PERFORMANCE}

In this study, to evaluate the heating uniformity performance of various feed structures, an evaluation index related to microwave is suggested. The heat generated in the dielectric is proportional to the power loss density $\left(\mathrm{W} / \mathrm{m}^{3}\right)$ due to electromagnetic waves expressed as

$$
P=\left(\sigma+\omega \varepsilon^{\prime \prime}\right)|\mathbf{E}|^{2},
$$

where $\sigma$ and $\varepsilon$ " represent the conductivity of the VOC absorbent dielectric medium and the imaginary part of the dielectric constant, respectively [16]. Although heat transfer should also be considered, only thermal losses due to microwaves are considered in this study. On the basis of the power loss density distribution, the figure of merit for performance evaluation is set as

$$
\sigma_{\text {nor }}(\mathbf{P})=\frac{\sqrt{\frac{\sum_{i=1}^{N}\left(P_{i}-\bar{P}\right)^{2}}{N-1}}}{\bar{P}},
$$

$N$ : Number of samples

$P_{i}$ : Power loss density at the $i$-th sample

$\bar{P}$ : Average value of power loss density

Table 3. Normalized shunt admittance values at $2.45 \mathrm{GHz}$ and slot's properties $\left(\mathrm{SAWFM}_{\text {cavity }}\right)$

\begin{tabular}{lccc}
\hline \multirow{2}{*}{ Slot } & \multirow{2}{*}{$\begin{array}{c}\text { Normalized } \\
\text { admittance }\end{array}$} & \multicolumn{2}{c}{ Properties $(\mathrm{mm})$} \\
\cline { 3 - 4 } & & Offset & Length \\
\hline 1 & $0.1017-j 0.0011$ & 7.2 & 58.4 \\
2 & $0.0987-j 0.0029$ & 7.5 & 59.4 \\
3 & $0.0981+j 0.0076$ & 8.6 & 58.2 \\
4 & $0.0993+j 0.0066$ & 9.2 & 60.5 \\
5 & $0.0990-j 0.0024$ & 9.0 & 58.8 \\
6 & $0.0982+j 0.0005$ & 7.0 & 59.1 \\
7 & $0.1027-j 0.0012$ & 8.8 & 58.5 \\
8 & $0.0992-j 0.0001$ & 9.5 & 59.4 \\
9 & $0.0966+j 0.0030$ & 9.5 & 58.8 \\
10 & $0.1018+j 0.0006$ & 7.7 & 59.4 \\
\hline
\end{tabular}

where $\sigma_{\text {nor }}(\mathbf{P})$ is the normalized standard deviation of power loss density in the material. To ensure the convergence of the calculation results, the field sampling interval is set to $7 \mathrm{~mm}$, which is smaller than $\lambda_{d} / 7$, where $\lambda_{d}$ is the wavelength in the dielectric medium. The total number of field samples in the dielectric is about 100,000 in this example. To evaluate the heating uniformity of horn-type and proposed systems, full wave EM simulation (CST Microwave Studio) is performed on the conventional horn-type feeding structure and the two WSA models $\left(\mathrm{SAWFM}_{\text {free }}\right.$ and $\left.\mathrm{SAWFM}_{\text {cavity }}\right)$.

Fig. 8 shows the power loss density distribution inside the dielectric medium. Fig. 8(a) and (b) show the results for the horn-type feeding structure. Fig. 8(c) and (d) are the results for SAWFM $_{\text {free, }}$ and Fig. 8(e) and (f) are the results for SAWFMcavity. It can be seen that the structure using the horn type has a strong power loss density distribution in the vicinity of the aperture. On the other hand, it can be seen that the power loss density distributions are relatively more uniform in the $\mathrm{SAWFM}_{\text {free }}$

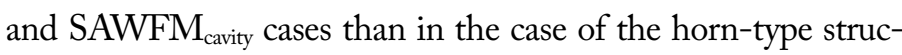
ture. The normalized standard deviations are given in Table 4. It is confirmed that the slotted waveguide feeding structure improves the heating uniformity by about $52 \%$ compared with the existing horn-type feeding structure. In addition, notice that there is little difference (less than 1\%) in heating uniformity between the two slotted waveguide models.

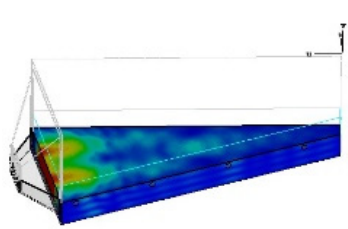

(a)

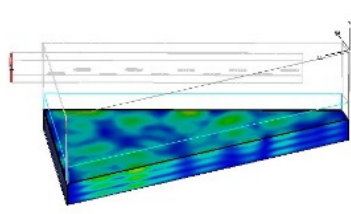

(c)

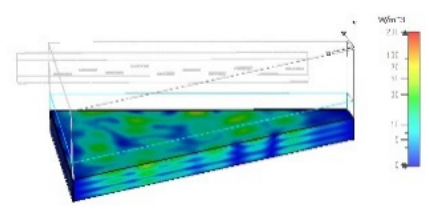

(e)

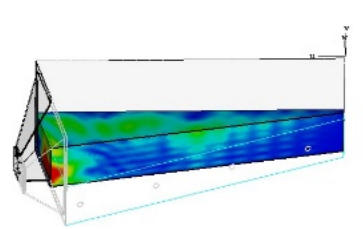

(b)
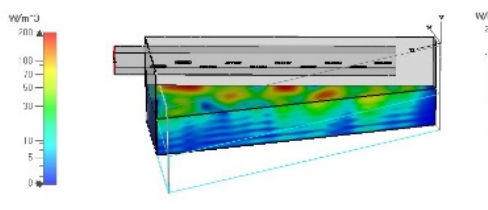

(d)

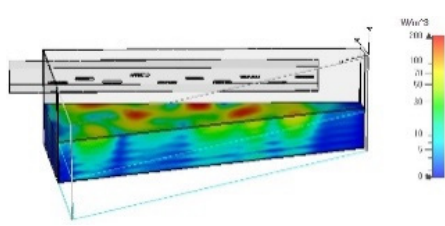

(f)
Fig. 8. Power loss density distribution in the material: (a) horn-type structure horizontal cut, (b) horn-type structure vertical cut, (c) SAWFM free $_{\text {structure horizontal cut, (d) SAWFM free }}$ structure vertical cut, (e) SAWFM cavity $_{\text {structure horizontal }}$

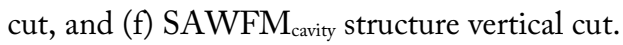


Table 4. Comparison of uniform heating performance

\begin{tabular}{lcc}
\hline Structure & $\begin{array}{c}\text { Average power loss } \\
\text { density }\left(\mathrm{W} / \mathrm{m}^{3}\right)\end{array}$ & $\begin{array}{c}\text { Normalized standard } \\
\text { deviation }\end{array}$ \\
\hline Horn type & 11.70 & 2.62 \\
$\mathrm{SAWFM}_{\text {free }}$ & 12.70 & 1.25 \\
$\mathrm{SAWFM}_{\text {cavity }}$ & 13.50 & 1.26 \\
\hline
\end{tabular}

\section{IMPEDANCE MATCHING CHARACTERISTICS}

Fig. 9 shows the impedance matching characteristics of the three systems. The reflection coefficient characteristic for $S(1,1)$ is usually required to be lower than $-10 \mathrm{~dB}$. The return loss is larger than $10 \mathrm{~dB}$ for both designs to satisfy the impedance matching requirement. However, in the case of heating facilities using microwaves, it is better to make the reflection as small as possible (for example, $S(1,1) \leq 13 \mathrm{~dB}$ corresponding to less than $5 \%$ reflection) for energy-efficient operation. To meet the conditions, an external matching circuit such as a waveguide stub tuner should be additionally attached for the horn type and

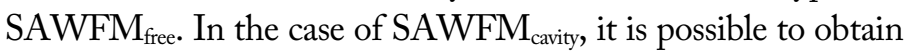
excellent impedance matching characteristics at a center frequency of $2.45 \mathrm{GHz}$ without additional impedance matching circuits. This is because a more accurate impedance matching is possible since the calculation of the normalized admittance value of each slot includes the cavity.

Generally, the resonance-based WSA antenna has very narrow band characteristics. Therefore, when it is used as the feeding structure in an industrial microwave heating facility, it may have characteristics that are very sensitive to internal load changes (e.g., dielectric constant, height, etc.). Even though the impedance matching can be obtained through the external matching circuit, the tuner has to be adjusted whenever the internal condition of the cavity changes.

Fig. 10(a) and (b) show the impedance matching characteristics according to the loading of VOC absorbent for $\mathrm{SAWFM}_{\text {free }}$ and

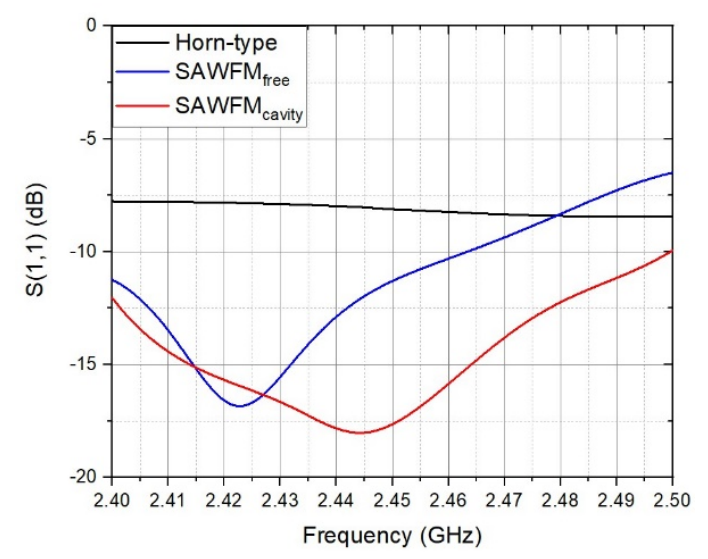

Fig. 9. Impedance matching characteristics.

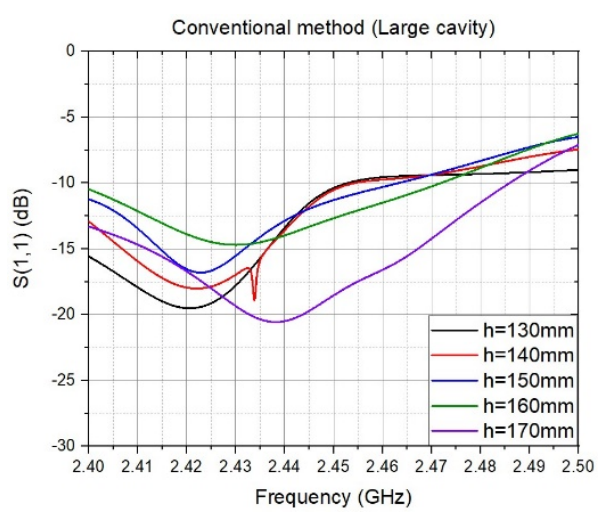

(a)

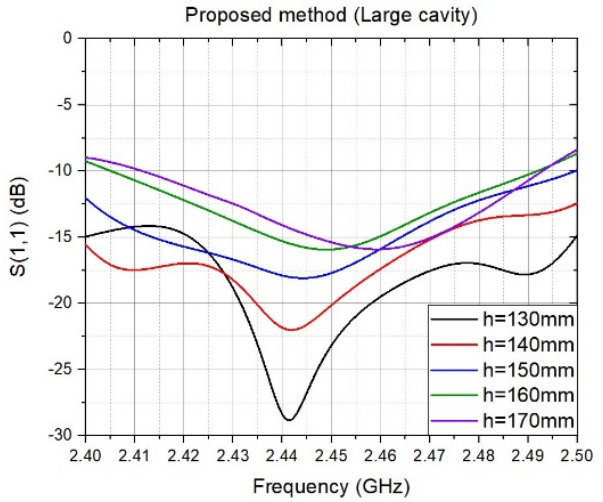

(b)

Fig. 10. Impedance matching characteristics with changing internal load condition (reference dielectric height $h=150 \mathrm{~mm}$ ): (a) $\mathrm{SAWFM}_{\text {free }}$ and (b) $\mathrm{SAWFM}_{\text {cavity. }}$.

$\mathrm{SAWFM}_{\text {caxity, }}$ respectively. The height of the VOC absorbent material inside the cavity varies from 130 to $170 \mathrm{~mm}$ with 10 $\mathrm{mm}$ intervals. In both models, the reference dielectric height is $150 \mathrm{~mm}$. In the case of $\mathrm{SAWFM}_{\text {free, }}$ it can be seen that the impedance matching characteristic deteriorates to $-10 \mathrm{~dB}$ depending on the change in dielectric height. However, in the case of SAWFM $_{\text {cavity, }}$ it can be seen that the impedance matching characteristic is maintained below $-15 \mathrm{~dB}$ despite the dielectric height change, and it can be stably operated without manipulating an external matching circuit according to a change of the load in the cavity.

\section{CONCLUSION}

In this study, two types of slotted waveguide array in the frequency of $2.45 \mathrm{GHz}$ are designed to improve the heating uniformity of the quadrangular prismatic microwave cavity used for a VOC removal system. Both follow the equivalent circuit approach of the WSA antenna design. The difference is whether the cavity is included when extracting the impedance data of the slot. Both methods confirm that the heating uniformity is improved by $52 \%$ compared with the conventional horn-type excitation case, and the uniformity difference between the two 
models is below than $1 \%$. However, the model using the impedance data of the slot including the cavity has been found to have better impedance matching by $6.35 \mathrm{~dB}$ and shows better loadinsensitive characteristics than the model designed by extracting the impedance data of the slot in the free space. As a result, the impedance value of the slot should be extracted taking the cavity effect into account for the design of the slotted waveguide feeding structure that improves the reflection and load-insensitive characteristics while improving the heating uniformity compared with the horn-type feeding structure.

This work was supported by Business for Cooperative R\&D between Industry, Academy, and Research Institute funded Korea Small and Medium Business Administration in 2018 and the BK21 Plus program of the Creative Research Engineer Development for IT, Seoul National University in 2020 (No. C0527861).

\section{REFERENCES}

[1] A. C. Metaxas, "Microwave heating," IET Power Engineering Journal, vol. 5, no. 5, pp. 237-247, 1991.

[2] J. M. Osepchuk, "A history of microwave heating applications," IEEE Transactions on Microwave Theory and Techniques, vol. 32, no. 9, pp. 1200-1224, 1984.

[3] R. J. Meredith, Engineers' Handbook of Industrial Microwave Heating. London, UK: Institution of Electrical Engineers, 1998.

[4] F. I. Khan and A. K. Ghoshal, "Removal of volatile organic compounds from polluted air," Journal of Loss Prevention in the Process Industries, vol. 13, no. 6, pp. 527-545, 2000.

[5] S. J. Yoon, J. S. Min, J. Y. Kim, S. J. Park, Y. K. Han, J. Y. Choi, N. U. Cho, and S. G. Moon, "System for removing VOCs using gas distribution plate," Korea Patent 102017-0105033, July 2017.

[6] D. Neumaier, S. Sanseverino, G. Link, and J. Jelonnek, "Homogeneous dielectric heating in large microwave ovens by excitation of multiple eigenmodes at their resonance frequencies," in Proceedings of the 17th International Confer- ence on Microwave and High Frequency Heating (AMPERE), Valencia, Spain, 2019, pp. 166-173.

[7] S. H. Bae, M. G. Jeong, J. H. Kim, and W. S. Lee, "A continuous power-controlled microwave belt drier improving heating uniformity," IEEE Microwave and Wireless Guided Wave Letters, vol. 27, no. 5, pp. 527-529, 2017.

[8] D. A. Hill, "Electronic mode stirring for reverberation chambers," IEEE Transactions on Electromagnetic Compatibility, vol. 36, no. 4, pp. 294-299, 1994.

[9] E. Dominguez-Tortajada, J. Monzo-Cabrera, and A. DiazMorcillo, "Uniform electric field distribution in microwave heating applicators by means of genetic algorithms optimization of dielectric multilayer structures," IEEE Transactions on Microwave Theory and Techniques, vol. 55, no. 1, pp. 85-91, 2007.

[10] V. Castrillo, G. D’Ambrosio, R. Massa, F. Chiadini, V. Fiumara, A. Scaglione, G. Panariello, and I. M. Pinto, "Improved design of waveguide slot array applicators for microwave heating," Materials Research Innovations, vol. 8, no. 2, pp. 71-74, 2004.

[11] A. A. Barba, D. Acierno, and M. d'Amore. "Use of microwaves for in-situ removal of pollutant compounds from solid matrices," Journal of Hazardous Materials, vol. 207, pp. 128-135, 2012.

[12] S. Ahn, C. Jeong, D. Lim, and W. Lee, "Kilowatt-level power-controlled microwave applicator with multiple slotted waveguides for improving heating uniformity," IEEE Transactions on Microwave Theory and Techniques, vol. 68, no. 7, pp. 2867-2875, 2020.

[13] R. E. Collin, Antennas and Radiowave Propagation. New York, NY: McGraw-Hill, 1985, pp. 265-273.

[14] A. F. Stevenson, "The theory of slots in rectangular waveguides," Journal of Applied Physics, vol. 19, no. 1, pp. 24-38, 1948.

[15] A. A. Oliner, "The impedance properties of narrow radiating slots in the broad face of rectangular waveguide: Part I. theory," IRE Transactions on Antennas and Propagation, vol. 5, no. 1, pp. 4-11, 1957.

[16] A. C. Metaxas, Industrial Microwave Heating. London, UK: Institution of Electrical Engineers, 1983, pp. 5-11. 


\section{Taewoo $\mathrm{Yu}$}

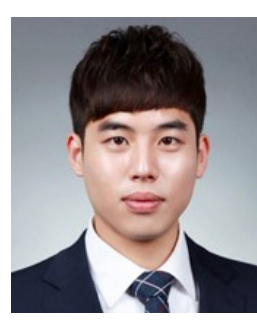

received his B.S. degree in electronic and electrical engineering from Hongik University, Seoul, Korea, in 2017. He is currently working toward an integrated master's and Ph.D. degree in the Department of Electrical and Computer Engineering at Seoul National University, Seoul, Korea. His main research interests are industrial microwave applicators and RF systems including wireless power transfer and radar systems.

\section{Hyunwook Lee}

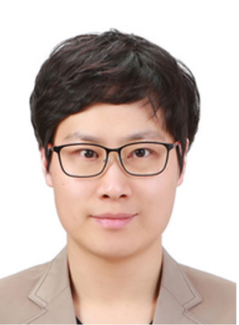

received his B.S., M.S., and Ph.D. degrees, all in radio sciences and engineering, from Kwangwoon University, Seoul, Korea, in 2007, 2009, and 2015, respectively. From 2015 to 2018, he was an assistant professor at the Center for Integrated Smart Sensors (CISS) at the Korea Advanced Institute of Science and Technology (KAIST), Daejeon, Korea. From 2018, he has been a researcher at ECOPRO Co. Ltd., Cheongju, Korea. His research interests are industrial microwave applicators including VOC removal and dehumidification systems.

\section{Sang-Jun Park}

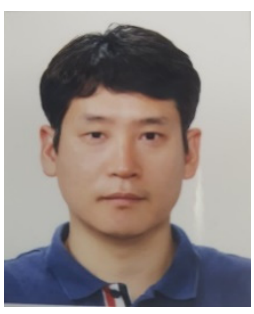

received his B.S. degree in chemical engineering from Donga University, Busan, Korea, in 2002, and M.S. degree in environmental engineering from Pohang University of Science and Technology in 2008. From 2012, he has been a researcher at ECOPRO Co. Ltd., Cheongju, Korea. Since 2018, he has been working toward Ph.D. degree in chemical engineering at Chungbuk University, Cheongju, Korea. His research interests are industrial microwave applicators including VOC removal and dehumidification systems.

\section{Sangwook Nam}

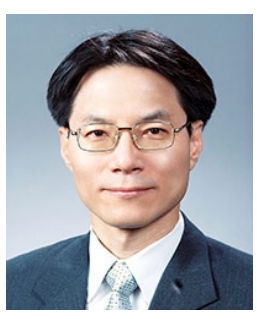

received his B.S. degree from Seoul National University, Seoul, Korea, in 1981, M.S. degree from Korea Advanced Institute of Science and Technology (KAIST), Seoul, Korea, in 1983, and Ph.D. degree from the University of Texas at Austin, Austin, TX, USA, in 1989, all in electrical engineering. From 1983 to 1986 , he was a researcher at the Gold Star Central Research Laboratory, Seoul, Korea. Since 1990, he has been a professor at the School of Electrical Engineering and Computer Science, Seoul National University. His research interests include analysis/design of electromagnetic structures, antennas, and microwave active/passive circuits. 